\title{
Quasi m-Cayley circulants
}

\author{
Ademir Hujdurović * \\ University of Primorska, FAMNIT, Glagoljaška 8, 6000 Koper, Slovenia \\ University of Primorska, IAM, Muzejski trg 2, 6000 Koper, Slovenia
}

Received 30 October 2011, accepted 4 February 2012, published online 15 June 2012

\begin{abstract}
A graph $\Gamma$ is called a quasi m-Cayley graph on a group $G$ if there exists a vertex $\infty \in V(\Gamma)$ and a subgroup $G$ of the vertex stabilizer $\operatorname{Aut}(\Gamma)_{\infty}$ of the vertex $\infty$ in the full automorphism group $\operatorname{Aut}(\Gamma)$ of $\Gamma$, such that $G$ acts semiregularly on $V(\Gamma) \backslash\{\infty\}$ with $m$ orbits. If the vertex $\infty$ is adjacent to only one orbit of $G$ on $V(\Gamma) \backslash\{\infty\}$, then $\Gamma$ is called a strongly quasi $m$-Cayley graph on $G$. In this paper complete classifications of quasi 2-Cayley, quasi 3-Cayley and strongly quasi 4-Cayley connected circulants are given.
\end{abstract}

Keywords: Arc-transitive, circulant, quasi m-Cayley graph.

Math. Subj. Class.: 05C15, 05C10

\section{Introduction}

Throughout this paper graphs are assumed to be finite, simple, connected and undirected, and groups are finite. Given a graph $\Gamma$ we let $V(\Gamma), E(\Gamma), A(\Gamma)$ and $\operatorname{Aut}(\Gamma)$ be the set of its vertices, edges, arcs and the automorphism group of $\Gamma$, respectively. A graph $\Gamma$ is said to be vertex-transitive, edge-transitive, and arc-transitive if its automorphism group acts transitively on $V(\Gamma), E(\Gamma)$ and $A(\Gamma)$, respectively.

Let $G$ be a finite group with identity element 1, and let $S \subset G \backslash\{1\}$ be such that $S^{-1}=S$. We define the Cayley graph $\operatorname{Cay}(G, S)$ on the group $G$ with respect to the connection set $S$ to be the graph with vertex set $G$, in which two vertices $x, y \in G$ are adjacent if and only if $x^{-1} y \in S$. A circulant of order $n$ is a Cayley graph on a cyclic group of order $n$.

In this paper we consider quasi-semiregular actions on graphs, a natural generalization of semiregular actions on graphs, which have been an active topic of research in the last decades (see, for example, [1, 2, 3, 4, 5, 8, 9, 11]). Following [7] we say that a group $G$ acts

*Supported in part by "Agencija za raziskovalno dejavnost Republike Slovenije”, research program P1-0285 and "Mladi raziskovalec" research program.

E-mail address: ademir.hujdurovic@upr.si (Ademir Hujdurović) 
quasi-semiregularly on a set $X$ if there exists an element $\infty$ in $X$ such that $G$ fixes $\infty$, and the stabilizer $G_{x}$ of any element $x \in X \backslash\{\infty\}$ is trivial. The element $\infty$ is called the point at infinity. A graph $\Gamma$ is called quasi $m$-Cayley on $G$ if the group $G$ acts quasi-semiregularly on $V(\Gamma)$ with $m$ orbits on $V(\Gamma) \backslash\{\infty\}$. If $G$ is cyclic and $m=1$ (respectively, $m=2$, $m=3$ and $m=4$ ) then $\Gamma$ is said to be quasi circulant (respectively, quasi bicirculant, quasi tricirculant and quasi tetracirculant). In addition, if the point at infinity $\infty$ is adjacent with only one orbit of $G_{\infty}$ then we say that $\Gamma$ is a strongly quasi $m$-Cayley graph on $G$.

Quasi $m$-Cayley graphs were first defined in 2011 by Kutnar, Malnič, Martinez and Marušič [7], who showed which strongly quasi $m$-Cayley graphs are strongly regular graphs.

In this paper, we consider which circulants are also quasi $m$-Cayley graphs. Our main results are stated in the following three theorems.

Theorem 1.1. Let $\Gamma$ be a quasi 2-Cayley graph of order $n$ which is also a connected circulant. Then either $\Gamma$ is isomorphic to the complete graph $K_{n}$, or $n \equiv 1(\bmod 4)$ is a prime and $\Gamma$ is isomorphic to the Paley graph $P(n)$. Moreover, $\Gamma$ is a quasi bicirculant.

Theorem 1.2. Let $\Gamma$ be a connected circulant. Then $\Gamma$ is also a quasi 3-Cayley graph if and only if either $\Gamma=K_{n}$, or replacing $\Gamma$ with its complement if necessary, $\Gamma \cong \operatorname{Cay}\left(\mathbb{Z}_{n}, S\right)$, where $S$ is the set of all non-zero cubes in $\mathbb{Z}_{n}$, and $n$ is a prime such that $n \equiv 1(\bmod 3)$. Moreover, $\Gamma$ is a quasi tricirculant.

Theorem 1.3. Let $\Gamma$ be a connected circulant. Then $\Gamma$ is a strongly quasi 4-Cayley graph on a group $G$ if and only if $\Gamma \cong C_{9}$ or $\Gamma \cong \operatorname{Cay}\left(\mathbb{Z}_{n}, S\right)$, where $S$ is the set of all fourth powers in $\mathbb{Z}_{n} \backslash\{0\}$, and $n$ is a prime such that $n \equiv 1(\bmod 4)$. Moreover, $\Gamma$ is a quasi tetracirculant.

The paper is organized as follows. In Section 2 we recall the classification of connected arc-transitive circulants. In Section 3 we prove Theorem 1.1 and in Section 4 we prove Theorems 1.2 and 1.3.

\section{Arc-transitive circulants}

We begin this section with the following lemma:

Lemma 2.1. Let $\Gamma$ be a connected vertex-transitive strongly quasi $m-$ Cayley graph. Then $\Gamma$ is arc-transitive.

Proof. Since $\Gamma$ is vertex transitive, it is sufficient to prove that there exists a vertex $v$ such that the stabilizer $\operatorname{Aut}(\Gamma)_{v}$ acts transitively on the neighborhood of $v$. It is obvious that if we choose the point at infinity for $v$, this condition is satisfied.

The previous lemma implies that we can somehow restrict our study to the connected arc-transitive circulants, therefore it is important to understand the structure of such graphs.

To state the classification of connected arc-transitive circulants, which has been obtained independently by Kovács [6] and Li [10], we need to recall certain graph products and the concept of normal Cayley graphs.

The wreath (lexicographic) product $\Sigma[\Gamma]$ of a graph $\Gamma$ by a graph $\Sigma$ is the graph with vertex set $V(\Sigma) \times V(\Gamma)$ such that $\left\{\left(u_{1}, u_{2}\right),\left(v_{1}, v_{2}\right)\right\}$ is an edge if and only if either $\left\{u_{1}, v_{1}\right\} \in E(\Sigma)$, or $u_{1}=v_{1}$ and $\left\{u_{2}, v_{2}\right\} \in E(\Gamma)$. For a positive integer $b$ and a graph 
$\Sigma$, denote by $b \Sigma$ the graph consisting of $b$ vertex-disjoint copies of the graph $\Sigma$. The graph $\Sigma\left[\overline{K_{b}}\right]-b \Sigma$ is called the deleted wreath (deleted lexicographic) product of $\Sigma$ and $\overline{K_{b}}$, where $\overline{K_{b}}=b K_{1}$.

Let $\Gamma=\operatorname{Cay}(G, S)$ be a Cayley graph on a group $G$. Denote by $\operatorname{Aut}(G, S)$ the set of all automorphisms of $G$ which fix $S$ setwise, that is,

$$
\operatorname{Aut}(G, S)=\left\{\sigma \in \operatorname{Aut}(G) \mid S^{\sigma}=S\right\} .
$$

It is easy to check that $\operatorname{Aut}(G, S)$ is a subgroup of $\operatorname{Aut}(\Gamma)$ and that it is contained in the stabilizer of the identity element $1 \in G$. It follows from the definition of Cayley graph that the left regular representation $G_{L}$ of $G$ induces a regular subgroup of $\operatorname{Aut}(\Gamma)$. Following $\mathrm{Xu}$ [12], $\Gamma=\operatorname{Cay}(G, S)$ is called a normal Cayley graph if $G_{L}$ is normal in $\operatorname{Aut}(\Gamma)$, that is, if $\operatorname{Aut}(G, S)$ coincides with the vertex stabilizer $1 \in G$. Moreover, if $\Gamma$ is a normal Cayley graph, then $\operatorname{Aut}(\Gamma)=G_{L} \rtimes \operatorname{Aut}(G, S)$.

Proposition 2.1. $[6,10]$ Let $\Gamma$ be a connected arc-transitive circulant of order $n$. Then one of the following holds:

(i) $\Gamma \cong K_{n}$;

(ii) $\Gamma=\Sigma\left[\bar{K}_{d}\right]$, where $n=m d, m, d>1$ and $\Sigma$ is a connected arc-transitive circulant of order $m$;

(iii) $\Gamma=\Sigma\left[\bar{K}_{d}\right]-d \Sigma$, where $n=m d, d>3, \operatorname{gcd}(d, m)=1$ and $\Sigma$ is a connected arc-transitive circulant of order $m$;

(iv) $\Gamma$ is a normal circulant.

In Section 3 and 4 two lemmas (that show that arc-transitive circulants described in Proposition 2.1(ii) and (iii) are not strongly quasi $k$-Cayley graphs) will be needed.

Lemma 2.2. Let $\Gamma$ be an arc-transitive circulant, described in Proposition 2.1(ii). Then $\Gamma$ is not a strongly quasi $k$-Cayley graph for any $k \in \mathbb{N}$.

Proof. We have $\Gamma=\Sigma\left[\bar{K}_{d}\right]$, where $n=m d, m, d>1$ and $\Sigma$ is a connected arc-transitive circulant of order $m$. Suppose that $\Gamma$ is a strongly quasi $k$-Cayley graph on a group $G$. Then $\operatorname{val}(\Gamma)=(n-1) / k=(m d-1) / k$. On the other hand, since $\Gamma=\Sigma\left[\bar{K}_{d}\right]$, we have $\operatorname{val}(\Gamma)=\operatorname{val}(\Sigma) \cdot d$. These two facts combined together imply that $d(m-k \cdot \operatorname{val}(\Sigma))=1$, and so $d=1$, a contradiction.

Lemma 2.3. Let $\Gamma$ be an arc-transitive circulant, described in Proposition 2.1(iii). Then $\Gamma$ is not a strongly quasi $k$-Cayley graph for any $k \in \mathbb{N}$.

Proof. We have $\Gamma=\Sigma\left[\bar{K}_{d}\right]-d \Sigma$, where $n=m d, d>3, \operatorname{gcd}(d, m)=1$, and $\Sigma$ is an arc-transitive circulant of order $m$. Suppose that $\Gamma$ is also a strongly quasi $k$-Cayley graph on a group $G$. By [10, Theorem 1.1] the $m$ copies of the graph $\overline{K_{d}}$ form an imprimitivity block system $\mathcal{B}$ for $\operatorname{Aut}(\Gamma)$. Clearly the block $B \in \mathcal{B}$ containing the point at infinity, that is, the trivial orbit of $G$, is fixed by $G$. This implies that $|G|$ divides $d-1$. On the other hand, since the valency of $\Gamma$ is $|G|$, we have $|G| \geq d-1$. Combining these results we obtain $|G|=d-1$. Thus, connectedness of $\Gamma$ implies that $m=2$. However, then there are $2 d-1$ vertices in $\Gamma$ different from the point at infinity, and they cannot be divided into $k$ orbits of size $d-1$ for any natural number $k$. Therefore, there are no strongly quasi $k$-Cayley graphs amongst the graphs from Proposition 2.1(iii) for any natural number $k \geq 1$. 
Lemma 2.4. Let $\Gamma$ be an arc-transitive circulant, described in Proposition 2.1(iv). If $\Gamma$ is also a strongly quasi $m$-Cayley graph on a group $G$, then the order of $\Gamma$ has at most $m+1$ divisors.

Proof. Let $\Gamma=\operatorname{Cay}\left(\mathbb{Z}_{n}, S\right)$ be a normal circulant. Let $A=\operatorname{Aut}(\Gamma)$. Since $\Gamma$ is a normal Cayley graph, $A \cong \mathbb{Z}_{n} \rtimes \operatorname{Aut}\left(\mathbb{Z}_{n}, S\right)$. We may, without loss of generality, assume that the point at infinity corresponds to the vertex $0 \in \mathbb{Z}_{n}$, and so $G \leq \operatorname{Aut}\left(\mathbb{Z}_{n}, S\right) \leq \operatorname{Aut}\left(\mathbb{Z}_{n}\right) \cong$ $\mathbb{Z}_{n}^{*}$. Therefore, $G \lesssim \mathbb{Z}_{n}^{*}$. Since $G$ has $m$ orbits on $\mathbb{Z}_{n} \backslash\{0\}$, then $\operatorname{Aut}\left(\mathbb{Z}_{n}\right)$ has at most $m$ orbits on $\mathbb{Z}_{n} \backslash\{0\}$, and at most $m+1$ orbits on $\mathbb{Z}_{n}$. Elements in the same orbit of $\operatorname{Aut}\left(\mathbb{Z}_{n}\right)$ are clearly of the same order in $\mathbb{Z}_{n}$. There exist an element in $\mathbb{Z}_{n}$ of order $d$, if and only if $d$ divides $n$. Therefore the number of divisors of $n$, denoted by $\tau(n)$, is not greater than $m+1$, i.e. $\tau(n) \leq m+1$.

\section{Quasi 2-Cayley graphs}

In this section the connected circulants are considered. In particular, connected circulants that are also quasi 2-Cayley graphs are classified (see Theorem 1.1). If a graph $\Gamma$ of order $n$ is a quasi 2-Cayley graph on a group $G$, which is not a strongly quasi 2-Cayley graph, then it is isomorphic to the complete graph $K_{n}$. Namely, in such a graph, the point at infinity $\infty$ is adjacent to both nontrivial orbits of $G$, and thus it is adjacent to all the vertices different from $\infty$. Consequently, we can conclude that $\Gamma$ has valency $|V(\Gamma)|-1$, and so $\Gamma$ is a complete graph. In order to classify all connected circulants that are also quasi 2-Cayley graphs it therefore suffices to characterize strongly quasi 2-Cayley graphs that are also connected circulants, we do this in Theorem 3.1.

Theorem 3.1. Let $\Gamma$ be a connected circulant. Then $\Gamma$ is also a strongly quasi 2-Cayley graph if and only if $\Gamma$ is isomorphic to the Paley graph $P(p)$, where $p$ is a prime such that $p \equiv 1(\bmod 4)$. Moreover, $\Gamma$ is a quasi bicirculant.

Proof. Let $\Gamma$ be the Paley graph $P(p)$, where $p$ is a prime, such that $p \equiv 1(\bmod 4)$. It is well known that the Paley graphs are connected arc-transitive circulants, and, as was observed in [7], they are also strongly quasi 2-Cayley graphs.

Conversely, let $\Gamma$ be a connected circulant $\operatorname{Cay}\left(\mathbb{Z}_{n}, S\right)$ of order $n$ not isomorphic to the complete graph $K_{n}$, which is also a strongly quasi 2-Cayley graph on a group $G$. Then $|G|=(n-1) / 2$ and $\Gamma$ is of valency $(n-1) / 2$. Lemma 2.1 tells us that $\Gamma$ is an arc-transitive graph, and moreover Proposition 2.1, Lemma 2.2 and Lemma 2.3 combined together imply that $\Gamma$ is a normal circulant. The theorem now follows from the three claims below.

Claim 1: $n$ is an odd prime.

It is obvious that $n$ must be odd, since 2 divides $n-1$. By Lemma 2.4 we have that $\tau(n) \leq 3$. Thus we have the following two possibilities for $n$ :

- $n=p$, where $p$ is a prime;

- $n=p^{2}$, where $p$ is a prime.

Suppose that the latter case hold. Let $A=\operatorname{Aut}(\Gamma)$. Since $\Gamma$ is a normal Cayley graph, we have $A \cong \mathbb{Z}_{n} \rtimes \operatorname{Aut}\left(\mathbb{Z}_{n}, S\right)$. We may, without loss of generality, assume that the point at infinity corresponds to the vertex $0 \in \mathbb{Z}_{n}$, and so $G \leq \operatorname{Aut}\left(\mathbb{Z}_{n}, S\right) \leq \operatorname{Aut}\left(\mathbb{Z}_{n}\right) \cong \mathbb{Z}_{n}^{*}$. Therefore, $\mathbb{Z}_{n}^{*}$ contains a subgroup $G$ of order $(n-1) / 2$. Since $\left|\mathbb{Z}_{n}^{*}\right| \leq n-1$ and $|G|$ 
divides $\left|\mathbb{Z}_{n}^{*}\right|$ we obtain that $\left|\mathbb{Z}_{n}^{*}\right|=n-1$ or $(n-1) / 2$. Since, by assumption, $n$ is not a prime, we have $\left|\mathbb{Z}_{n}^{*}\right|=(n-1) / 2$. This gives in the following equation

$$
\frac{p^{2}-1}{2}=p(p-1)
$$

which has the unique solution $p=1$, a contradiction.

CLAIM 2: $n \equiv 1(\bmod 4)$.

Since $S=-S$, and no element in $\mathbb{Z}_{n}$ can be its own inverse, we have that the number of elements in $S$ is even, and since $|S|=\frac{n-1}{2}$, we have $n \equiv 1(\bmod 4)$.

Claim 3: $\Gamma$ is isomorphic to the Paley graph $P(n)$.

By Claim $1, n$ is a prime. Therefore the group $\mathbb{Z}_{n}^{*}$ is cyclic, and thus since $G$ is a subgroup of $\mathbb{Z}_{n}^{*}, G$ is cyclic as well. By [6, Remark 2], we have Aut $(\Gamma)=\left\{g \mapsto g^{\sigma}+h \mid \sigma \in\right.$ $\left.K, h \in \mathbb{Z}_{n}\right\}$, for a suitable group $K<\operatorname{Aut}\left(\mathbb{Z}_{n}\right)$, and $S$ is the orbit under $K$ of a generating element of $\mathbb{Z}_{n}$, that is, $S=\operatorname{Orb}_{K}(g)$ for some generating element $g$ of $\mathbb{Z}_{n}$. Now we have that $\operatorname{Aut}(\Gamma)_{0}=\left\{g \mapsto g^{\sigma}+h \mid \sigma \in K, h \in \mathbb{Z}_{n}: 0^{\sigma}+h=0\right\}=\left\{g \mapsto g^{\sigma} \mid \sigma \in K\right\} \cong K$. So we see that $G \lesssim K$. Since $S=\operatorname{Orb}_{K}(g) \lesssim \operatorname{Orb}_{G}(g)$, and $|S|=\left|\operatorname{Orb}_{G}(g)\right|$ we have that $S \cong \operatorname{Orb}_{G}(g)$, which gives us that $S \cong G$ (taking $g=1$ ). Now, since $G$ is the index 2 subgroup of the cyclic group $\mathbb{Z}_{n}^{*}, G$ is of the form $G=\left\langle x^{2}\right\rangle$ where $x$ generates $\mathbb{Z}_{n}^{*}$. Therefore $G$ consists of all squares in $\mathbb{Z}_{n}^{*}$ and $S \cong G$, implying that $\Gamma$ is isomorphic to the Paley graph $P(n)$ as claimed.

It is obvious that $G$ must be cyclic, so the graph $\Gamma$ is in fact a quasi bicirculant.

Proof of Theorem 1.1: It follows from Theorem 3.1 and the paragraph preceding it.

In general, if $\Gamma$ is a vertex transitive quasi 2-Cayley graph on a group $G$, not isomorphic to the complete graph, then it is a strongly regular graph of a rank 3 group. Namely, the orbits of $G$ are contained in the orbits of the stabilizer of the $\operatorname{Aut}(\Gamma)_{\infty}$ and since there are just two nontrivial orbits of $G$, then there are exactly two nontrivial orbits of the $\operatorname{Aut}(\Gamma)_{\infty}$ which in fact must coincide with the orbits of $G$. Therefore Aut $(\Gamma)$ must be a rank 3-group, and the graphs of the rank 3 groups are strongly regular graphs.

\section{Quasi 3-Cayley and 4-Cayley graphs}

In this section we will deal with the question which connected circulants are also quasi 3-Cayley graph or strongly quasi 4-Cayley graphs. We first consider the case of strongly quasi 3-Cayley graphs.

Theorem 4.1. Let $\Gamma$ be a connected circulant. Then $\Gamma$ is also a strongly quasi 3-Cayley if and only if $\Gamma \cong \operatorname{Cay}\left(\mathbb{Z}_{n}, S\right)$ where $S$ is the set of all non-zero cubes in $\mathbb{Z}_{n}$, and $n$ is a prime such that $n \equiv 1(\bmod 3)$. Moreover, $\Gamma$ is a quasi tricirculant.

Proof. Let $\Gamma=\operatorname{Cay}\left(\mathbb{Z}_{p}, S\right)$ where $p \equiv 1(\bmod 3)$ is a prime and $S$ is the set of all nonzero cubes in $\mathbb{Z}_{p}$. Since $p$ is a prime, it is well known that $\operatorname{Aut}\left(\mathbb{Z}_{p}\right) \cong \mathbb{Z}_{p}^{*}$ is a cyclic group of order $p-1$. Let $G=\left\langle a^{3}\right\rangle$, where $a$ is a generating element of $\mathbb{Z}_{p}^{*}$. Then $G$ consists of all non-zero cubes in $\mathbb{Z}_{p}$, and $|G|=\frac{p-1}{3}$. The action of $G$ on $\mathbb{Z}_{p}$ defined by $x^{g}=g \cdot x$ gives $G$ as the subgroup of $\operatorname{Aut}(\Gamma)$. The group $G$ acts quasi-semiregularly on $\mathbb{Z}_{p}$ with $0 \in \mathbb{Z}_{p}$ 
as the point at infinity. Namely, it is easy to check that $G_{0}=G$, and that the stabilizer of any element $x \in \mathbb{Z}_{p} \backslash\{0\}$ is trivial. Since $|G|=\frac{p-1}{3}$, it follows that $G$ has 3 orbits on $\mathbb{Z}_{p} \backslash\{0\}$, and therefore $\Gamma$ is a quasi 3-Cayley graph. Since one of the orbits of $G$ is the set $S$, the point at infinity is adjacent to only one orbit of $G$, so $\Gamma$ is in fact a strongly quasi 3-Cayley graph. By the construction $\Gamma$ is an arc-transitive circulant since $G \leq \operatorname{Aut}(\Gamma)_{0}$ acts transitively on the set of vertices adjacent to the vertex 0 .

Conversely, let $\Gamma$ be a connected circulant of order $n$, which is also a strongly quasi 3-Cayley graph on a group $G$. Then $|G|=\frac{n-1}{3}$. From Lemma 2.1 we have that $\Gamma$ is arctransitive, and therefore Proposition 2.1, Lemma 2.2 and Lemma 2.3 combined together imply that $\Gamma$ is a normal circulant. Therefore, we can assume that $\Gamma=\operatorname{Cay}\left(\mathbb{Z}_{n}, S\right)$, and that $G \leq \operatorname{Aut}\left(\mathbb{Z}_{n}, S\right) \leq \operatorname{Aut}\left(\mathbb{Z}_{n}\right)$, implying that $\frac{n-1}{3} \mid \varphi(n)$, where $\varphi(n)$ is the Euler totient function.

ClAiM 1: $n$ is a prime number.

Let

$$
n=p_{1}^{k_{1}} \cdot p_{2}^{k_{2}} \cdots p_{t}^{k_{t}}
$$

be a canonic factorization of a positive integer $n$. From Lemma 2.4, we have $\tau(n) \leq 4$. Now we can calculate

$$
\tau(n)=\left(k_{1}+1\right)\left(k_{2}+1\right) \cdots\left(k_{t}+1\right) .
$$

We have the following possibilities for $n$ :

- $n=p$,

- $n=p^{2}$;

- $n=p^{3}$;

- $n=p q$,

where $p$ and $q$ are different primes.

If $n=p^{2}$, then the only solution of $\frac{n-1}{3} \mid \varphi(n)$ is $p=2$ and $n=4$. However, if $n=4$, the graph $\Gamma$ is of valency 1 , so it is not a connected graph.

If $n=p^{3}$, then there is no solution of the above equation.

If $n$ is a product of two different primes, then we have $\left|\mathbb{Z}_{n}^{*}\right|=(n-1) / 3$ or $2(n-1) / 3$. In the first case $\mathbb{Z}_{n}^{*} \cong G$, so $\mathbb{Z}_{n}^{*}$ acts semiregularly on $\mathbb{Z}_{n} \backslash\{0\}$, and it is not difficult to see that this is not the case for $n=p q$. If $\left|\mathbb{Z}_{n}^{*}\right|=2(n-1) / 3$, then we obtain the following equation

$$
(p-1)(q-1)=\frac{2(p q-1)}{3} .
$$

The only solutions in natural numbers of the above equation are $(p, q) \in\{(4,7),(5,5)$, $(7,4)\}$, so there are no two different primes $p, q$ satisfying the given equation.

Having in mind all the written above, we conclude that $n$ is a prime.

Claim 2: $\Gamma$ is isomorphic to the Cayley Graph $\operatorname{Cay}\left(\mathbb{Z}_{n}, S\right)$, where $S$ is set of all non zero cubes in $\mathbb{Z}_{n}$, and $n$ is a prime such that $n \equiv 1(\bmod 3)$.

Similarly as in the previous section, it can be shown that $G \cong S$. Since $G$ is an index 3 subgroup of $\mathbb{Z}_{n}^{*}$, we have $G=\left\langle x^{3}\right\rangle$, where $x$ is a generating element of $\mathbb{Z}_{n}^{*}$. It follows that $G$ consists of all cubes in $\mathbb{Z}_{n}^{*}$, so $\Gamma$ is isomorphic to $\operatorname{Cay}\left(\mathbb{Z}_{n}, S\right)$, where $S$ is the set of all non zero cubes in $\mathbb{Z}_{n}$ and $n \equiv 1(\bmod 3)$ is a prime. It is obvious from the mentioned above, that the group $G$ must be cyclic, therefore, $\Gamma$ is in fact a quasi tricirculant. 
Proof of the Theorem 1.2: Let $\Gamma$ be a connected circulant of order $n$, which is also a quasi 3-Cayley on a group $G$. The point at infinity is adjacent to all three nontrivial orbits of $G$, if and only if $\Gamma$ is isomorphic to $K_{n}$. If the point at infinity is adjacent to just one nontrivial orbit of $G$, then $\Gamma$ is a strongly quasi 3-Cayley graph, therefore, Theorem 4.1 gives us the desired result. If the point at infinity is adjacent to two nontrivial orbits of $G$, then we consider the complement $\Sigma=\bar{\Gamma}$ of the graph $\Gamma$. The graph $\Sigma$ is a quasi 3-Cayley graph on $G$, and actually it is a strongly quasi 3-Cayley graph on $G$. Since $\Sigma$ is the complement of a circulant it is also a circulant. Suppose that $\Sigma$ is not connected. Then, since it is vertex-transitive, it is the disjoint union of some isomorphic graphs. The point at infinity is adjacent to one orbit of $G$, so the connected components of $\Sigma$ must have at least $1+\frac{n-1}{3}$ points. Therefore $n=k \cdot n_{1}$, where $k$ is the number of connected components, and $n_{1}$ is the number of points in each of the components. We have noticed that $n_{1} \geq 1+\frac{n-1}{3}$, thus $k \leq 2$. If $k=1$ then $\Sigma$ is connected. Suppose that $k=2$. Then there are two connected components of $\Gamma$, say $\Gamma_{1}$ and $\Gamma_{2}$, each containing $n / 2$ points. Suppose that $\infty \in \Gamma_{1}$. Let $\Delta_{1}, \Delta_{2}$ and $\Delta_{3}$ be a nontrivial orbits of $G$, and let the point at infinity be adjacent to $\Delta_{1}$. Then $\Delta_{1} \subset \Gamma_{1}$. Since $\Gamma_{1}$ and $\Gamma_{2}$ have the same size, it means that at least one of $\Delta_{2}$ and $\Delta_{3}$ have points both in $\Gamma_{1}$ and $\Gamma_{2}$. Suppose that $u, v \in \Delta_{2}$, such that $u \in \Gamma_{1}$ and $v \in \Gamma_{2}$. Since $u$ and $v$ are in the same orbit of $G$ then there exist $g \in G$ which maps $u$ to $v$. However, $g$ fixes $\infty$, and consequently $g$ fixes $\Gamma_{1}$, a contradiction.

Having in mind all the written above, we see that $\Sigma$ is a connected circulant, which is also a strongly quasi 3-Cayley graph. Therefore we have the desired result.

We will continue this section with the proof of Theorem 1.3.

Proof of Theorem 1.3: Let $\Gamma=C_{9}$. Then $\Gamma \cong \operatorname{Cay}\left(\mathbb{Z}_{9},\{ \pm 1\}\right)$. Then the group $G=$ $\{1,-1\} \subset \mathbb{Z}_{9}^{*}$ acts quasi semiregularly on $\mathbb{Z}_{9}$ with 0 as the point at infinity.

Let $\Gamma \cong \operatorname{Cay}\left(\mathbb{Z}_{p}, S\right)$, where $S$ is the set of all fourth powers in $\mathbb{Z}_{p} \backslash\{0\}$, and $p$ is a prime such that $p \equiv 1(\bmod 4)$. Define $G=\left\langle a^{4}\right\rangle$, where $a$ is some generating element of $\mathbb{Z}_{p}^{*}$, which is cyclic in this case. We have that $G$ acts quasi-semiregularly on $\mathbb{Z}_{p}^{*}$ with 0 as the point at infinity. Since $|G|=\frac{p-1}{4}$, it follows that $G$ has 4 orbits on $\mathbb{Z}_{p} \backslash\{0\}$, and therefore $\Gamma$ is a quasi 4-Cayley graph. It is also easy to see that 0 is adjacent to only one orbit of $G$ on $\mathbb{Z}_{p} \backslash\{0\}$, therefore $\Gamma$ is a strongly quasi 4-Cayley graph. By the construction, $\Gamma$ is a connected arc-transitive circulant.

Conversely, let $\Gamma$ be a connected circulant of order $n$ which is also a strongly quasi 4-Cayley graph on a group $G$. Then $|G|=(n-1) / 4$. Using Lemma 2.1 we have that $\Gamma$ is arc-transitive, and so Proposition 2.1, Lemma 2.2 and Lemma 2.3 combined together imply that $\Gamma$ is a normal circulant. Therefore, we can assume that $\Gamma=\operatorname{Cay}\left(\mathbb{Z}_{n}, S\right)$, and that $G \leq \operatorname{Aut}\left(\mathbb{Z}_{n}, S\right) \leq \operatorname{Aut}\left(\mathbb{Z}_{n}\right)$, implying that

$$
\frac{n-1}{4} \mid \varphi(n)
$$

Using Lemma 2.4 we obtain $\tau(n) \leq 5$. So we have the following possibilities:

- $n=p$,

- $n=p^{2}$,

- $n=p^{3}$,

- $n=p^{4}$,

- $n=p q$, 
where $p$ and $q$ are different primes.

If $n=p^{2}$, then the only solution of (4.1) is $n=9$. In this case, the valency of $\Gamma$ is $(9-1) / 4=2$, so $\Gamma \cong C_{9}$.

In the cases when $n=p^{3}$, and $n=p^{4}$ there is no prime satisfying (4.1).

When $n=p q$, we have that $(p-1)(q-1)=\alpha \cdot(p q-1) / 4$, where $\alpha \in\{1,2,3\}$. If $\alpha=1$, then we have $\mathbb{Z}_{n}^{*}=G$, so $\mathbb{Z}_{n}^{*}$ must act semiregularly on $\mathbb{Z}_{n} \backslash\{0\}$, which is not the case. If $\alpha=2$, then there are no two different primes satisfying $(p-1)(q-1)=$ $(p q-1) / 2$, and finally, when $\alpha=3$, we have that $n=5 \cdot 13$ is the only possibility. In this case, $\Gamma$ is a connected arc-transitive circulant on 65 vertices, which has valency 16. Since $G$ is an index 3 subgroup of $\mathbb{Z}_{65}^{*} \cong \mathbb{Z}_{4} \times \mathbb{Z}_{12}$, then we can calculate $G \cong\{ \pm 1, \pm 8, \pm 12, \pm 14, \pm 18, \pm 21, \pm 27, \pm 31\}$, and we can see that $G$ does not act semiregularly on $\mathbb{Z}_{65} \backslash\{0\}$. Namely, the non identity element $21 \in G$ fixes the point $13 \in \mathbb{Z}_{65} \backslash\{0\}$.

Assume now that $n$ is a prime. Similarly as in the proof of Theorem 3.1, we obtain $G \cong S$, and therefore, since $G$ is an index 4 subgroup of $\mathbb{Z}_{n}^{*}$, we have $G=\left\langle x^{4}\right\rangle$, where $x$ is some generating element of $\mathbb{Z}_{n}^{*}$. Therefore, $\Gamma \cong \operatorname{Cay}\left(\mathbb{Z}_{n}, S\right)$, where $S$ is the set of all fourth powers in $\mathbb{Z}_{n} \backslash\{0\}$.

From the mentioned above, it is clear that $G$ is a cyclic group, so $\Gamma$ is in fact a quasi tetracirculant.

\section{References}

[1] E. Dobson, A. Malnič, D. Marušič and L. A. Nowitz, Minimal normal subgroups of transitive permutation groups of square-free degree, Discrete Math. 307 (2007), 373-385.

[2] E. Dobson, A. Malnič, D. Marušič and L. A. Nowitz, Semiregular automorphisms of vertextransitive graphs of certain valencies. J. Combin. Theory Ser. B 97 (2007), 371-380.

[3] M. Giudici, Quasiprimitive groups with no fixed point free elements of prime order, J. London Math. Soc. 67 (2003), 73-84.

[4] M. Giudici, New constructions of groups without semiregular subgroups, Comm. Algebra 35 (2007), 2719-2730.

[5] M. Giudici and J. Xu, All vertex-transitive locally-quasiprimitive graphs have a semiregular automorphism, J. Algebr. Combin. 25 (2007), 217-232.

[6] I. Kovács, Classifying arc-transitive circulants, J. Algebr. Combin. 20 (2004), 353-358.

[7] K. Kutnar, A. Malnič, L. Martinez and D. Marušič, Quasi $m$-Cayley strongly regular graphs, manuscript.

[8] K. Kutnar and D. Marušič, Recent trends and future directions in vertex-transitive graphs, Ars Math Contemp. 1 (2008), 112-125.

[9] K. Kutnar and P. Šparl, Distance-transitive graphs admit semiregular automorphisms, European J. Combin. 31 (2010), 25-28.

[10] C. H. Li, Permutation groups with a cyclic regular subgroup and arc-transitive circulants, $J$. Algebr. Combin. 21 (2005), 131-136.

[11] D. Marušič, On vertex symmetric digraphs, Discrete Math. 36 (1981), 69-81.

[12] M. Y. Xu, Automorphism groups and isomorphisms of Cayley digraphs, Discrete Math. 182 (1998), 309-320. 